ARTICLE

\title{
Shear band-driven precipitate dispersion for ultrastrong ductile medium-entropy alloys
}

\author{
Tae Jin Jang ${ }^{1,6}$, Won Seok Choi ${ }^{2,6}$, Dae Woong Kim³ ${ }^{3}$ Gwanghyo Choi ${ }^{2}$, Hosun Jun (1) ${ }^{2}$, Alberto Ferrari(D) ${ }^{4}$, \\ Fritz Körmann ${ }^{4,5}$, Pyuck-Pa Choi (iD) ${ }^{2 凶} \&$ Seok Su Sohn (i) ${ }^{1 凶}$
}

Precipitation strengthening has been the basis of physical metallurgy since more than 100 years owing to its excellent strengthening effects. This approach generally employs coherent and nano-sized precipitates, as incoherent precipitates energetically become coarse due to their incompatibility with matrix and provide a negligible strengthening effect or even cause brittleness. Here we propose a shear band-driven dispersion of nano-sized and semicoherent precipitates, which show significant strengthening effects. We add aluminum to a model CoNiV medium-entropy alloy with a face-centered cubic structure to form the L2 ${ }_{1}$ Heusler phase with an ordered body-centered cubic structure, as predicted by ab initio calculations. Micro-shear bands act as heterogeneous nucleation sites and generate finely dispersed intragranular precipitates with a semicoherent interface, which leads to a remarkable strength-ductility balance. This work suggests that the structurally dissimilar precipitates, which are generally avoided in conventional alloys, can be a useful design concept in developing high-strength ductile structural materials.

\footnotetext{
${ }^{1}$ Department of Materials Science and Engineering Korea University, Seoul, South Korea. ${ }^{2}$ Department of Materials Science and Engineering Korea Advanced Institute of Science and Technology, Daejeon, South Korea. ${ }^{3}$ Center for High Entropy Alloys Pohang University of Science and Technology, Pohang, South Korea. ${ }^{4}$ Department of Materials Science and Engineering Delft University of Technology, Mekelweg 2, Delft, The Netherlands. ${ }^{5}$ Max-Planck-Institut für Eisenforschung Max-Planck-Straße 1, Düsseldorf, Germany. ${ }^{6}$ These authors contributed equally: Tae Jin Jang and Won Seok Choi. ${ }^{凶}$ email: p.choi@kaist.ac.kr; sssohn@korea.ac.kr
} 
S triving for developing materials with ultrahigh strengths and sufficient uniform ductility has been an imperative challenge in structural applications. The aim has been usually achieved by configuring a disordered matrix responsible for ductility with ordered phases as precipitates to hinder dislocation motion. This so-called precipitation strengthening, especially when introducing structurally similar second phases coherent to the matrix, is remarkably effective in restraining the sacrifice of ductility ${ }^{1-3}$. The coherency enables the precipitates to distribute homogeneously in nanoscale, resulting in coherency strain fields and antiphase-boundary strengthening, as for instance in multicomponent Co-based or Ni-based superalloys consisting of a $\mathrm{Ll}_{2}$ ordered face-centered-cubic (FCC) phase in a disordered FCC matrix ${ }^{4}$. However, excessive alloying of the ordered phase-forming elements may lead to the formation of phases structurally dissimilar to the matrix called topologically closed-packed (TCP) phases such as the $\sigma$ phase, the $\mu$ phase, and the Laves phases, resulting in the inhomogeneous distribution at grain boundaries at a coarse microscale due to a loss of coherency ${ }^{5-7}$. This microstructure makes the alloys prone to catastrophic failure in load-bearing applications. Consequently, the incoherent precipitates have been perceived as detrimental, to be suppressed by delicate controls of alloying elements and thermomechanical treatments ${ }^{8-10}$.

Nevertheless, to conquer the severe brittleness of dissimilar phases with a matrix, one feasible way is to manipulate semicoherent precipitates by avoiding the formation of incoherent interfaces with the matrix. The lower interfacial energy of semicoherent precipitates than incoherent ones reduces their coarsening, while the homogeneous distribution in nanoscale can be embodied by introducing additional nucleation sites in the lattice, as reported in low-density steel and $\mathrm{Al}$ alloys ${ }^{11,12}$. To implement our design philosophy and develop ultrastrong alloys with good ductility, we choose an equiatomic ternary $\mathrm{Co}-\mathrm{Ni}-\mathrm{V}$ alloy as a model matrix system. This medium-entropy alloy (MEA), as a subclass of multiprincipal element alloys (MPEAs) or highentropy alloys (HEAs) possessing single-phase structure ${ }^{13-15}$, exhibits great mechanical properties, in particular a yield strength of $\sim 1 \mathrm{GPa}$, attributed to severe lattice distortion, and a tensile ductility of $38 \%{ }^{15}$. This property is ascribed from only solidsolution and grain-boundary strengthening at an average $\sim 2 \mu \mathrm{m}$ grain size in FCC-structured matrix. However, it is challenging to further enhance the mechanical properties of $\mathrm{CoNiV}$ alloys as an additional refinement of grains is restricted in conventional processing due to the limited process windows ${ }^{16}$. In this respect, precipitation strengthening can be an attractive candidate for further improving the mechanical properties. Recently, unceasing endeavors have been exerted in developing MPEAs through precipitation strengthening, and several studies demonstrated that homogenously distributed $\mathrm{L}_{2}$ nanoparticles in a FCC matrix are particularly effective in significantly enhancing strength while retaining moderate ductility ${ }^{17-22}$. Most of the studies exploited coherent precipitates, but there was no attempt yet to achieve improved properties by adopting semicoherent precipitates dissimilar to the matrix ${ }^{23-25}$.

Here we present a CoNiV-based MEA that can be strengthened through the formation of semicoherent nanoprecipitates and thermomechanical treatments enabling them to disperse homogeneously in the lattice. To realize such a material, $\sim 6.25$ at $\% \mathrm{Al}$ is added to form an $\mathrm{L} 2{ }_{1}$ ordered body-centered-cubic (BCC) phase in a FCC matrix, based on density-functional theory (DFT) calculations. A conventional cold-rolling process is conducted to introduce sufficient lattice defects into the material, and a subsequent heat treatment promoted the formation of precipitates and the recrystallization, assisted by the stored energy near the lattice defects. The material processes tailoring the size and morphology of the precipitates, with the aid of the high dislocation density, allow for effective strengthening, leading to a remarkable strength-ductility balance. Our approach demonstrates that structurally dissimilar precipitates, which are generally avoided due to their negligible strengthening or detrimental effect on ductility, can provide a useful design concept for the development of high-strength ductile structural materials.

\section{Results}

Material and process design. Aluminum is one of the most widely used elements in HEAs and MEAs as secondary phase former. The ordered phases that can be generated by the sole addition of $\mathrm{Al}$ or combined with $\mathrm{Ti}$ are the $\mathrm{B} 2(\mathrm{CoAl}, \mathrm{NiAl})$ and $\mathrm{Ll}_{2}\left(\mathrm{Co}_{3} \mathrm{Al}, \mathrm{Ni}_{3} \mathrm{Al}\right)$ phases for Co-Ni-containing FCC solidsolution alloys, such as $\mathrm{CoCrNi}$, $\mathrm{CoCrFeNi}, \mathrm{CoCrCuFeNi}$, or $\mathrm{CoCrFeMnNi}{ }^{19,26-29}$. In this study, we added $\mathrm{Al}$ to the $\mathrm{CoNiV}$ MEA, which showed enhanced mechanical properties imputed to severe lattice distortion ${ }^{15}$. DFT calculations (Fig. 1a) determined the most stable precipitate as a Co-rich $\mathrm{L} 2{ }_{1}$ phase among the ordered $\mathrm{L}_{2}-(\mathrm{Co}, \mathrm{Ni})_{3} \mathrm{Al}, \mathrm{B} 2-(\mathrm{Co}, \mathrm{Ni}) \mathrm{Al}$, and $\mathrm{L}_{1}-(\mathrm{Co}, \mathrm{Ni})_{2} \mathrm{VAl}$ phases, indicating that the high concentration of $\mathrm{V}$ affects the stability of ordered phases. The stabilization of this phase originates from the opening of a pseudogap in the electronic density of states of the L2 1 phase (Fig. 1b) in proximity to the Fermi level $\left(E_{F}\right)$, which is a common indication of a compound with high formation energy because more electronic states can be accommodated at lower energies. As for the stoichiometry of this L2 phase, we note that the larger the Co concentration, the closer the pseudogap to $E_{F}$, signaling increased stability of the $\mathrm{L}_{1}$ phase with an increasing amount of Co in this precipitate (see Supplementary Note for detailed information).

Figure $1 \mathrm{c}$ displays the overall schematics of the thermomechanical processing to fabricate the precipitation-strengthened $\mathrm{Al}_{0,2} \mathrm{CoNiV}$ alloys. As predicted, the homogenized state consisted of a coarse FCC matrix and Al-rich L2 ${ }_{1}$ islands (Supplementary Fig. 1), in which the cold-rolling process-induced macroscopic and microscopic shear bands (Supplementary Fig. 2). The macroshear bands were formed during cold-rolling and consequent strain localization, resulting in thick lines distributed at $\sim 10-45^{\circ}$ to the rolling direction. These macroshear bands do not need to follow crystallographic orientations. On the other hand, micro-shear bands were developed inside the FCC grains alongside the macroshear bands. It is known that the microshear bands can be in the form of twins bundles, dense stackingfault bundles, or linear arrays of dislocations and thus they are aligned along specific crystallographic shear planes $^{30}$. After subsequent recrystallization and aging processes $\left(1150{ }^{\circ} \mathrm{C}\right.$ for $2 \mathrm{~h}$ and $850{ }^{\circ} \mathrm{C}$ for $1 \mathrm{~h}$, respectively), referred to as RA, the $\mathrm{L} 22_{1}$ phase was also observed in the form of islands and particles at grain boundaries or triple junctions (Supplementary Fig. 3). These $\mathrm{L} 2{ }_{1}$ precipitates at grain boundaries do not significantly contribute to strengthening because it is difficult for dislocations to directly interact with precipitates during glides, but they rather readily pile up in front of precipitates at the pre-existing pile-up sites, i.e., the grain boundaries. Instead, here we utilized the abundant micro-shear bands as nucleation sites via partial recrystallization treatment for obtaining nanosized precipitates finely dispersed inside grains. Therefore, the heat-treatment conditions were selected to be 800,850 , and $900^{\circ} \mathrm{C}$ for $1 \mathrm{~h}$ to control the recrystallization and precipitation behavior.

Microstructural characterization. The MEA designed in this work (V: 31.25, Co: 31.25, Ni: 31.25, Al: 6.25 (in at\%) or $\mathrm{Al}_{0.2} \mathrm{CoNiV}$ ), heat-treated at three different temperatures, are hereafter referred to as A800, A850, and A900, respectively (see 

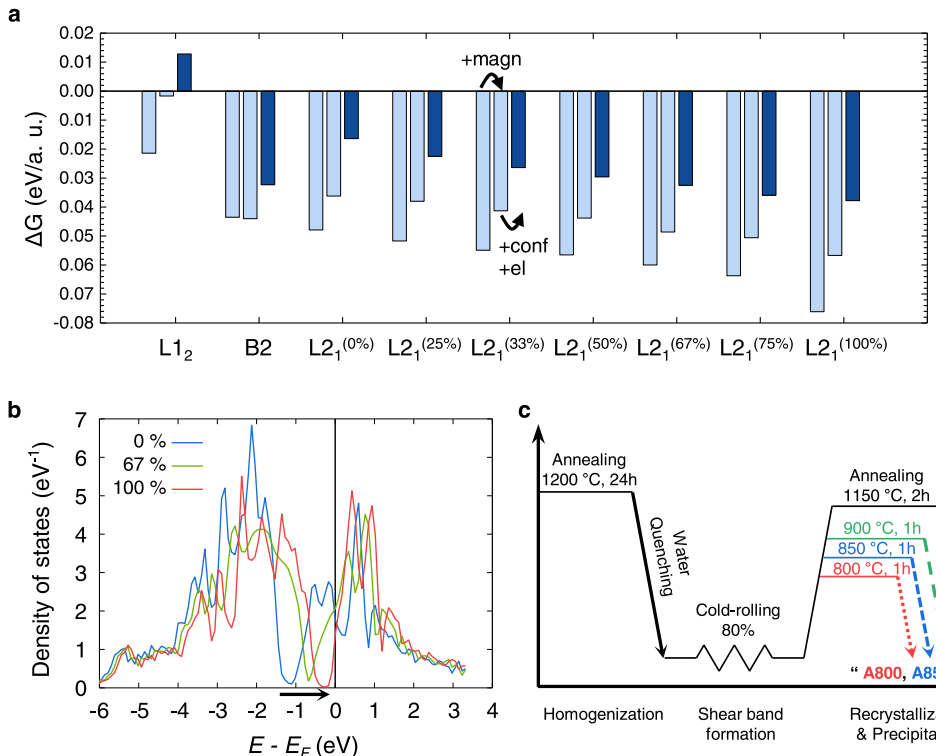

c

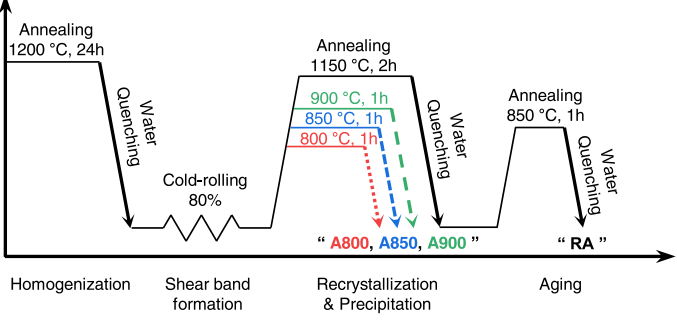

$\mathbf{L 2}_{1}$

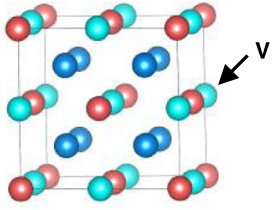

d

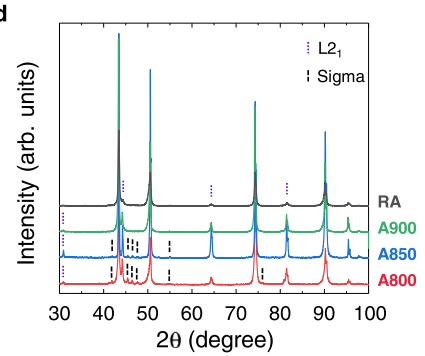

Fig. 1 Material and process design. a Free energy of the candidate precipitates with respect to the solid solution. The percentages for the $L 2{ }_{1}$ phase indicate the occupation of the $(\mathrm{Co}, \mathrm{Ni})$ sublattice by $\mathrm{Co}$. For each phase, the first, second, and third bars represent the $0 \mathrm{~K}\left(-273^{\circ} \mathrm{C}\right.$ ) energy difference in the ferromagnetic state, the $0 \mathrm{~K}\left(-273^{\circ} \mathrm{C}\right)$ energy difference in the paramagnetic state, and the free energy difference at $1150 \mathrm{~K}\left(877{ }^{\circ} \mathrm{C}\right)$ with the inclusion of configurational and electronic entropy, respectively. $\mathbf{b}$ Densities of states of the $\mathrm{L} 2{ }_{1}$ phase for different Co contents in the ferromagnetic state. c Schematics of the thermomechanical processing for precipitation-strengthened $\mathrm{Al}_{0.2}$ CoNiV alloys. d Phase identification via X-ray diffraction (XRD) analysis of the annealed $\mathrm{Al}_{0.2} \mathrm{CoNiV}$ alloys.

"Methods"). As shown by X-ray diffraction (XRD) (Fig. 1d), the primary peaks of the alloys corresponded to the FCC phase, while the secondary peaks were superlattice reflections stemming from the $\mathrm{L} 2{ }_{1}$-ordered phase. The A850 and A800 alloys additionally contained the $\sigma$ phase, present for the most part in the $\mathrm{L} 2{ }_{1}$ islands and with a smaller fraction also in the nearby recrystallized FCC grains (Supplementary Fig. 4). Figure 2a-f shows electron backscatter diffraction (EBSD) maps for the investigated alloys, revealing FCC (Fig. 2a-c) and $\mathrm{L}_{1}$ (Fig. 2d-f) phases. For A800 (Fig. 2a), the FCC grains show a bimodal size distribution resulting from partial recrystallization. The recrystallized FCC grains have an average size of $\sim 1 \mu \mathrm{m}$, and are distributed along macroshear bands formed at $\sim 45^{\circ}$ to the cold-rolling direction. This bimodal distribution can be attributed to the high stored energy in the shear bands, which promote heterogeneous nucleation. The coarse FCC grains presented in the ashomogenized state are refined by macroscopic shear bands induced from cold-rolling, leading to an average grain size of $212.1 \pm 73.4 \mu \mathrm{m}$. The $\mathrm{L} 2{ }_{1}$ grains also show a bimodal size distribution, with a size of $7.4 \mu \mathrm{m}$ for the coarse island and a few hundred nanometers for the fine particles (Fig. 2d). Electronchanneling contrast imaging (ECCI) shows the microstructure of the present alloys more precisely (Fig. $2 \mathrm{~g}-\mathrm{i}$ ). For A800, the L 21 particles exhibit a fine rod-like shape with an average diameter of $\sim 57 \mathrm{~nm}$ and aspect ratio of $\sim 3$, and they are aligned along the micro-shear bands inside the non-recrystallized FCC matrix. These $\mathrm{L} 2{ }_{1}$ nanoparticles have Kurdjumov-Sachs (K-S) orientation relationship with the FCC matrix, which will be discussed later in this report. It is noteworthy that very fine additional FCC laths are observed inside the $\mathrm{L} 2{ }_{1}$ islands, having a width of $\sim 40 \mathrm{~nm}$ and interspacing of $\sim 29 \mathrm{~nm}$.

As the heat-treatment temperature increases, the fraction and size of the recrystallized FCC grains increase, resulting in a completely recrystallized structure in the A900 alloy (Fig. 2a-c). $\mathrm{L} 2{ }_{1}$ precipitates start to form at the grain boundaries or triple junctions with recrystallization process. These intergranular L $2_{1}$ particles exhibit an incoherent or only one-sided semicoherent interface with the adjacent disordered FCC grains (Supplementary Fig. 5). For the fully recrystallized sample (A900), the L2 particles at grain boundaries or triple junctions in the recrystallized FCC region are $\sim 320 \mathrm{~nm}$ in size. The $\mathrm{L}_{1}$ precipitates are also present inside the recrystallized FCC grains as intragranular particles. These intragranular $\mathrm{L} 22_{1}$ particles show an average size of $\sim 146 \mathrm{~nm}$. The FCC laths grow to $\sim 250 \mathrm{~nm}$ and the $\sigma$ phase disappears. The fractions of the constituent phases and their sizes are summarized in Supplementary Tables 1 and 2, respectively. Figure $2 \mathrm{j}, \mathrm{k}$ displays the overall microstructural evolutions of $\mathrm{Al}_{0.2} \mathrm{CoNiV}$ alloys.

Figure $3 a-d$ shows a detailed EBSD analysis of the A800 sample. The recrystallized FCC grains are located at the interface between the non-recrystallized FCC and $\mathrm{L} 22_{1}$ islands. This preferred nucleation behavior of FCC grains can be attributed to the strain incompatibility between the soft FCC phase and the hard $\mathrm{L} 22_{1}$ island, which can be confirmed by the high kernel average misorientation (KAM) values at their interfaces (Fig. 3b). Compared to the dislocation-free recrystallized grains, the non-recrystallized FCC grains show evident green lines along the $\{111\}$ plane trace indicating a high dislocation density $\left(1.77 \times 10^{15} \mathrm{~m}^{-2}\right)$ of non-recrystallized grains. This result demonstrates that the cold-rolling leads to the micro-shear bands along specific crystallographic $\{111\}$ planes as well as to macroshear bands. The crystallographic orientation of FCC laths is almost identical in each $\mathrm{L} 22_{1}$ island, implying that the two phases have well-defined orientation relationships (Supplementary Fig. 5).

The role of micro-shear bands on the $\mathrm{L} 2{ }_{1}$ formation is revealed in Fig. 3e-h. An ECCI map shows that the $\mathrm{L} 2{ }_{1}$ particles are well aligned along the micro-shear bands in a non-recrystallized FCC grain of the A800 alloy (Fig. 3e). Notably, distinctively contrasting line features along the micro-shear band are apparent, as indicated by the arrows. A transmission electron microscopy (TEM) image (Fig. 3f-h) shows such a precipitate region and an 


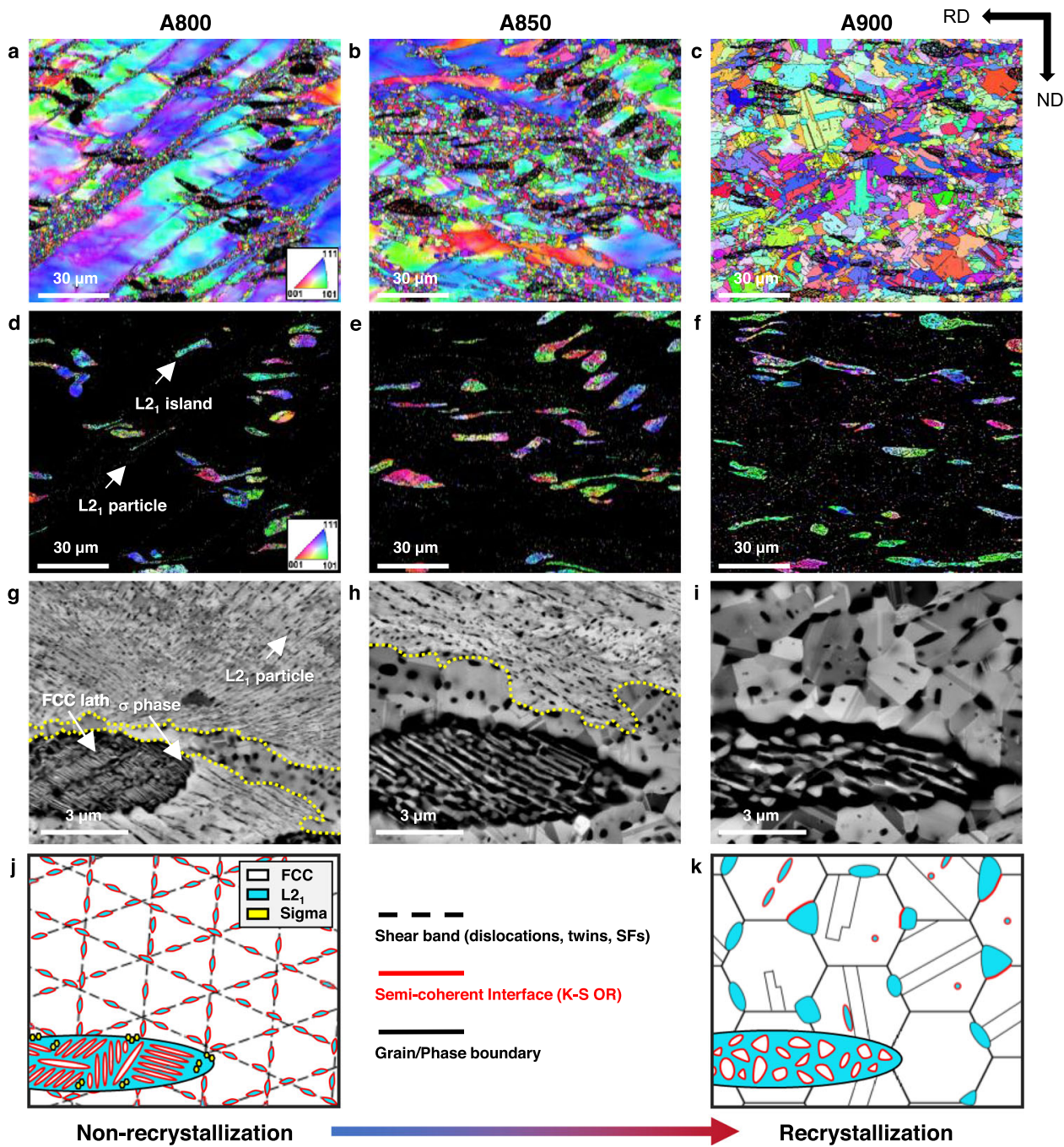

Fig. 2 Microstructural evolutions with increased heat-treatment temperature. a-c Electron backscatter diffraction (EBSD) maps of face-centered-cubic (FCC), $\mathbf{d}$-f EBSD maps of $\mathrm{L} 2_{2}$ phases, $\mathbf{g}$-i electron-channeling contrast imaging (ECCI) micrographs for $\mathrm{Al}_{0.2} \mathrm{CoNiV}$ alloys annealed at 800,850 , and $900^{\circ} \mathrm{C}$ for $1 \mathrm{~h}$. Yellow-dotted lines indicate the boundaries between the recrystallized region and the non-recrystallized region. $\mathbf{j}$, $\mathbf{k}$ Schematics showing the microstructural evolutions from non-recrystallized state to recrystallized state.

array of $\mathrm{L} 2{ }_{1}$ particles along the micro-shear bands. The observation of the FCC $(\gamma)$ and $\mathrm{L} 2{ }_{1}$ interface identifies a K-S orientation relationship between them $\left((111)_{\mathrm{FCC}} / /(1 \overline{1} 0)_{\mathrm{BCC}}\right.$, $\left.[\overline{1} 10]_{\mathrm{FCC}} / /[111]_{\mathrm{BCC}}\right)$ (Supplementary Fig. 6). High-resolution TEM and fast Fourier-transform (FFT) images confirm that the micro-shear bands consist of stacking faults (SFs) and nanotwin (NT) bundles (Fig. 3g, h). Figure 3i shows an atom probe tomography (APT) reconstruction acquired from the nonrecrystallized grain, which includes both FCC matrix and an L2 1 precipitate. An 1-D concentration profile across these two phases reveals that $\mathrm{Al}$ partitions to $\mathrm{L} 2{ }_{1}$, while $\mathrm{V}$ and $\mathrm{Ni}$ partition to FCC, and Co shows no pronounced partitioning. The measured chemical compositions of the FCC matrix and $\mathrm{L}_{2}$ were $40 \mathrm{~V}-34 \mathrm{Co}-24 \mathrm{Ni}-2 \mathrm{Al}($ at $\%)$ and $33 \mathrm{~V}-33 \mathrm{Co}-18 \mathrm{Ni}-16 \mathrm{Al}$ (at $\%$ ), respectively. Consequently, the precipitates are identified as the $(\mathrm{Co}, \mathrm{Ni})_{2}$ VAl-type $\mathrm{L}_{1}$, so-called Heusler phase.

Tensile properties. Figure $4 \mathrm{a}$ shows the room-temperature engineering stress-strain curves of our designed MEAs. To emphasize the substantial enhancement in mechanical properties upon the formation of the semicoherent nanoprecipitates, the curve of reference RA sample without the assistance of shear bands is also presented for comparison. The tensile properties are summarized in Supplementary Table 3. The fully recrystallized A900 alloy exhibits a yield strength of $1050 \pm 20 \mathrm{MPa}$, an ultimate tensile strength of $1480 \pm 11 \mathrm{MPa}$, and a total elongation of $31.7 \pm 4.4 \%$, similar to those of the CoNiV alloy with a grain size of $\sim 2 \mu \mathrm{m}^{15}$. This indicates that the $\mathrm{L} 2_{1}$ particles at grain boundaries have little effect on the yield strength. The small decrease in ductility by $6.3 \%$ can be attributed to ease of void nucleation and coalescence at interfaces between the rigid $\mathrm{L} 2{ }_{1}$ particles and soft FCC matrix ${ }^{31}$, because the brittle fracture was not observed (Supplementary Fig. 7). For the A800 alloy, the yield and ultimate tensile strengths are as high as $1500 \pm 15 \mathrm{MPa}$ and $1727 \pm 22 \mathrm{MPa}$, respectively. Most notably, we observe a remarkable strength-ductility balance of $1587 \mathrm{MPa} \times 26.7 \%$ for the A850 alloy. These remarkable properties are compared in Fig. $4 \mathrm{~b}$, $\mathrm{c}$ with other solid-solution or precipitation-strengthened HEAs and MEAs (Supplementary Table 4 for detailed information). The alloys designed in this work exhibit an excellent combination of ultrahigh strength and uniform ductility, surpassing the previously reported HEAs and MEAs having semicoherent and incoherent phases, and being comparable to the recently reported ultrastrong and ductile coherent $\mathrm{Ll}_{2}$ precipitation-strengthened HEAs and MEAs. The mechanical 
a

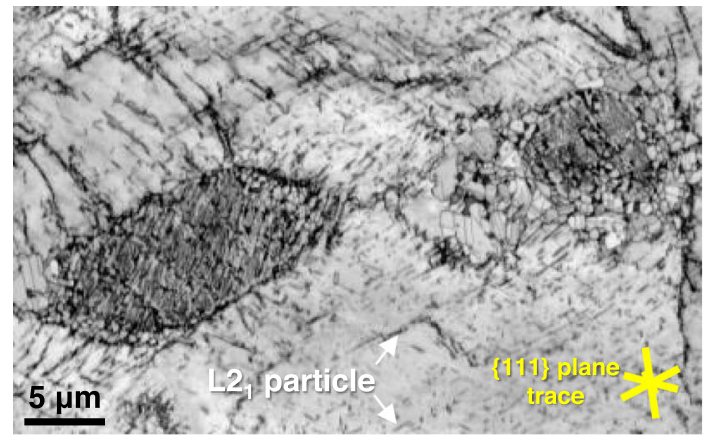

C

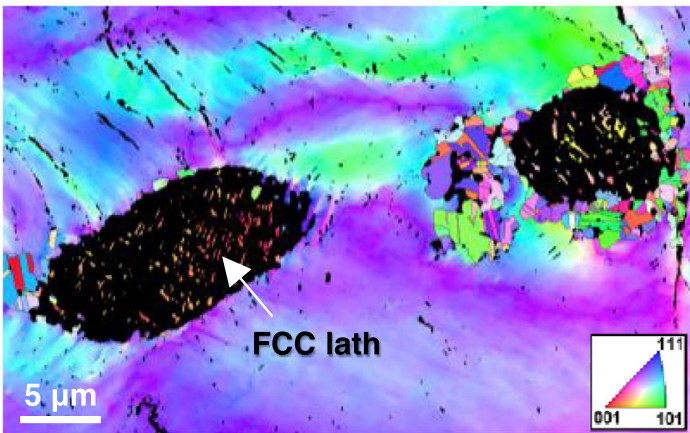

b

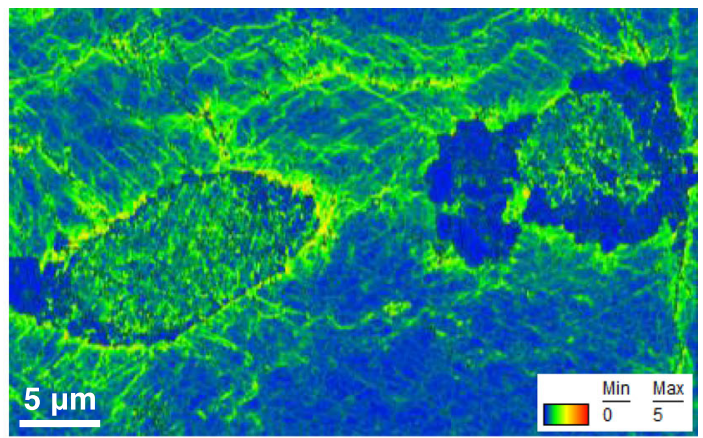

d

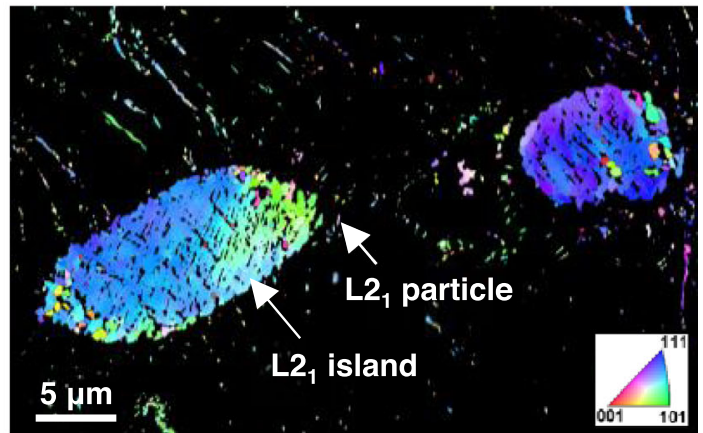

e

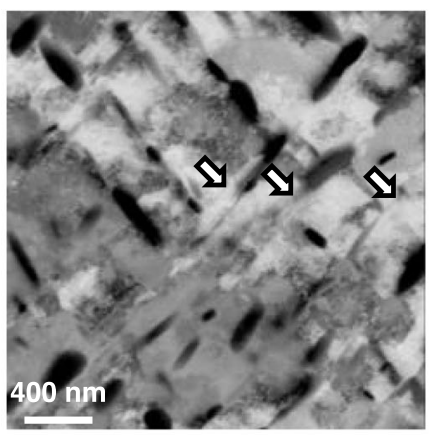

g

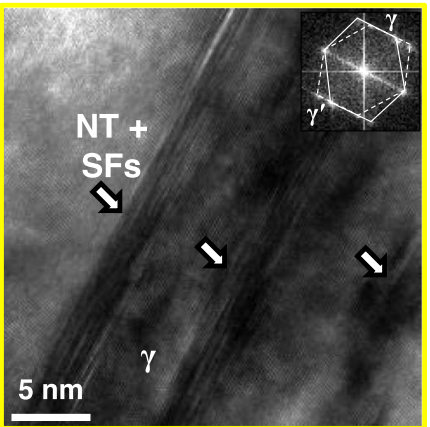

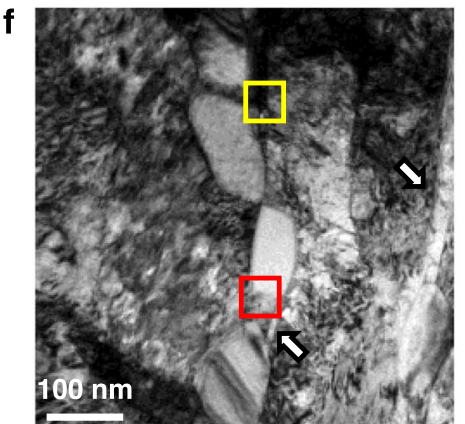

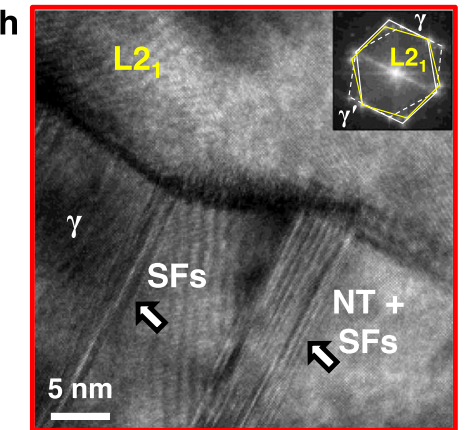

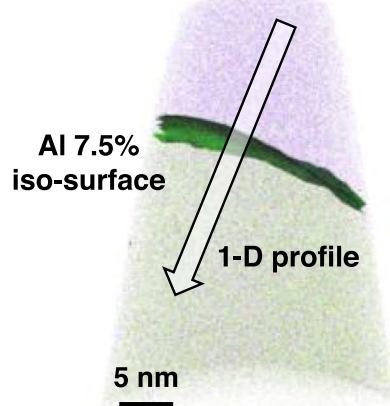

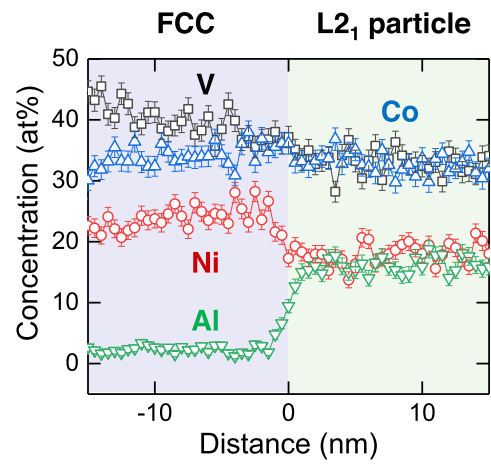

Fig. 3 The microstructure evolution in the medium-entropy $\mathbf{A l}_{\mathbf{0 . 2}} \mathbf{C o N i V}$ alloy heat-treated at $800^{\circ} \mathbf{C}$ for $\mathbf{1} \mathbf{h}$. a Electron backscatter diffraction (EBSD) images showing quality (IQ), b kernel average misorientation (KAM), inverse pole figure (IPF) maps of $\mathbf{c}$ face-centered-cubic (FCC) and $\mathbf{d}$ L2 $2_{1}$ phases. e Electron-channeling contrast imaging (ECCI) map, $\mathbf{f}$ transmission electron microscopy (TEM) image of micro-shear bands and $L 2_{1}$ particles in the nonrecrystallized FCC grains. $\mathbf{g}$, $\mathbf{h}$ High-resolution TEM and corresponding fast Fourier-transform (FFT) images indicate the micro-shear band consists of stacking faults and nanotwins. i Atom probe tomography (APT) tip reconstruction and 1-D profile across the $L 2_{1}$ and FCC matrix. The phase boundary is highlighted by a 7.5 at\% Al iso-concentration surface. Each datum point stands for an average concentration measured at a $0.5 \mathrm{~nm}$ interval, where error bars indicate a standard deviation.

properties of the present alloys seem to follow a linear trend of the strength-ductility combination of the CoNiV alloys. It should be noted that it is challenging to further enhance the mechanical properties of CoNiV alloys, as the limited process windows in conventional processing restrict the refinement of grains to sizes smaller than $\sim 2 \mu \mathrm{m}^{16}$. Another strengthening mechanism should be embodied and the present approach enables further improvement in mechanical properties. 
a

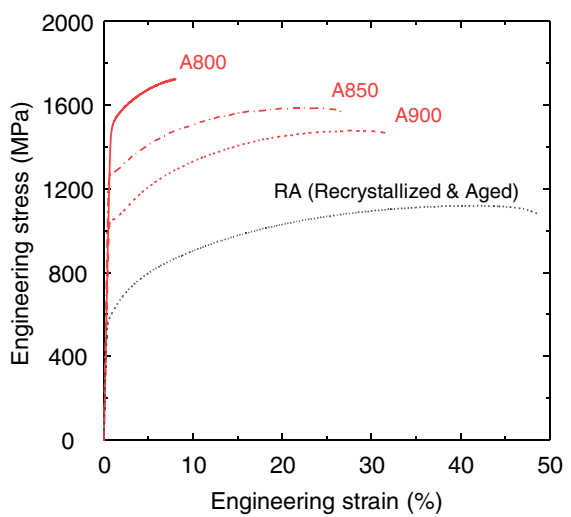

b

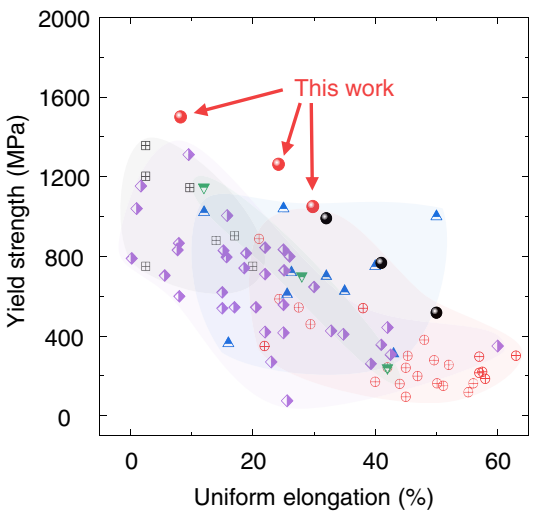

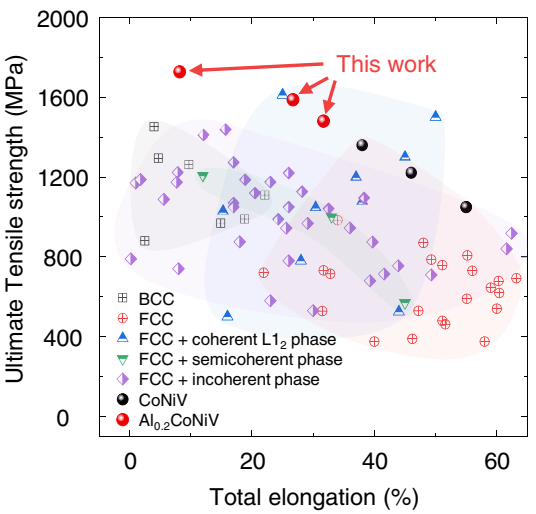

Fig. 4 Room-temperature mechanical properties of our alloys. a Engineering tensile stress-strain curves for the annealed $\mathrm{Al}_{0.2} \mathrm{CoNiV}$ alloys. $\mathbf{b}$ Overview of yield strength versus uniform elongation, and $\mathbf{c}$ Ultimate tensile strength versus total elongation values for the current $\mathrm{Al}_{0.2} \mathrm{CoNiV}$ alloy, compared to single or multiphase high-/medium-entropy alloys. The mechanical properties are summarized in Supplementary Table 3.

\section{Discussion}

Similar to the precipitation of the $\mathrm{L1}_{2}$-ordered FCC phase in the disordered FCC matrix, the $\mathrm{L}_{1}$ Heusler phase exhibits a high degree of coherency with the disordered BCC matrix ${ }^{32}$. However, in the present $\mathrm{Al}_{0.2} \mathrm{CoNiV}$ alloy, $\mathrm{V}$ promotes the formation of $\mathrm{L} 2{ }_{1}$ ordered BCC precipitates in a disordered FCC matrix. The L2 Heusler phase forms an incoherent interface with the matrix, and thus nucleates at grain boundaries or triple junctions. In addition, intergranular $\mathrm{L} 2{ }_{1}$ precipitates grow rapidly as the incoherent interface migrates at a high rate, thereby forming micron-sized coarse particles ${ }^{18}$. In this respect, the coherency between precipitates and the matrix plays a significant role in the formation and stability of uniformly and finely dispersed nanoprecipitates. The formation of homogeneously distributed fine nanoparticles is the key strategy for obtaining ultrastrong ductile precipitationstrengthened alloys.

Even though the FCC and $\mathrm{L}_{2}$ phases usually form an incoherent interface, they can form a semicoherent interface according to the $\mathrm{K}-\mathrm{S}$ or Nishiyama-Wassermann $(\mathrm{N}-\mathrm{W})$ orientation relationships between FCC and BCC structures. For both relationships, the closest packed planes of each structure are parallel to each other, i.e., $\{111\}_{\mathrm{FCC}} / /\{110\}_{\mathrm{BCC}}$, which correspond to their slip planes. This orientation indicates that $\mathrm{L} 22_{1}$ precipitates, nucleated at the slip plane of FCC, prefer to form a semicoherent interface having $\mathrm{K}-\mathrm{S}$ or $\mathrm{N}-\mathrm{W}$ relationships. In conjunction with the manipulation of orientation relationships, a strategy to avoid intergranular precipitates is to provide additional nucleation sites by introducing micro-shear bands inside the grains. The coldrolled and partially recrystallized alloy has a high dislocation density along the micro-shear bands in the non-recrystallized grains. As a wall of a high dislocation density shows higher energy than the lattice, it can act as a heterogeneous nucleation site. Moreover, the elastic field around the dislocation core lowers the activation energy of atomic migration and promotes pipediffusion and precipitation at dislocations ${ }^{33}$. Thus, the easy and profound nucleation of the $\mathrm{L} 22_{1}$ phase is achieved along $\{111\}$ planes containing deformation-induced defects, which cannot be commonly seen in other types of precipitate-containing alloys.

As stated above, the intergranular $\mathrm{L} 2{ }_{1}$ particles of the A900 alloy exhibit an incoherent or only one-sided semicoherent interface with the adjacent disordered FCC grains. In contrast, although not all the interfaces are indexed entirely due to the limited spatial resolution, the intragranular $\mathrm{L} 2{ }_{1}$ precipitates in the A800 alloy show a semicoherent interface on all sides having a K-S relationship with the disordered FCC matrix (Supplementary Fig. 5a). In addition, the intragranular $\mathrm{L} 2{ }_{1}$ precipitates are well aligned along the $\{111\}$ plane traces of FCC, corresponding to the slip planes. A semicoherent interface shows a low driving force for migration due to low interface energy and also relatively low mobility compared to incoherent interfaces, thus suppressing the growth of precipitates. In addition to the difference in the type of interfaces, the location of the precipitates contributes to their size difference. As shown in Supplementary Fig. 5b, the precipitates at grain boundaries in the A900 alloy with a recrystallized microstructure have a larger size than the precipitates inside the grains. The intergranular precipitates have direct access to the grain boundaries that could act as easy diffusion paths assisting the rapid growth of intergranular precipitates. Accordingly, intragranular L2 1 precipitates along the micro-shear bands become finer, with an average size of $\sim 60 \mathrm{~nm}$, as compared to intergranular precipitates at the grain boundaries, and they are distributed homogeneously inside grains due to micro-shear bands and partial recrystallization. Therefore, micro-shear bands play a key role in the nucleation of nanosized precipitates finely dispersed inside non-recrystallized grains, while macroshear bands promote the formation of fine recrystallized grains and intergranular L2 1 particles.

The current A800 and A850 alloys exhibit superb mechanical properties with a high-yield strength of 1262 and $1500 \mathrm{MPa}$, respectively. From the above microstructural observations, the $\mathrm{L} 2{ }_{1}$ precipitates significantly contribute to the strengthening, but the amount of this strengthening should be quantified because the high dislocation density also plays a dominant role in the enhancement of strength. For the A800 alloy, the strengthening contributions from solid solution, grain boundary, dislocation, and precipitation strengthening are calculated to be $\sim 383, \sim 359$, $\sim 293$, and $\sim 350 \mathrm{MPa}$, respectively ("Methods" and Supplementary Fig. 8). The calculated total yield strength is $1384 \mathrm{MPa}$, in reasonable agreement with the experimentally determined yield strength of $1500 \mathrm{MPa}$. The nanosized intragranular $\mathrm{L} 2_{1}$ precipitates combined with high dislocation density lead to remarkable strengthening increments for the A800 and A850 alloys by $25.3 \%$ and $11.2 \%$, respectively, compared to the A900 alloy.

Another feature of the designed alloy is that a promising uniform elongation of $\sim 8 \%$ is still achieved even at the strongly strengthened state. The semicoherent interfaces were enabled through the K-S orientation relationship, which lowers the interfacial energy and also promotes anisotropic growth along $\{111\}$ planes forming an aspect ratio of 3.03 and 2.83 for the A800 and A850 alloys, respectively ${ }^{34}$. Thus, according to the effective precipitate radius ahead of gliding dislocations, two characteristic 
a

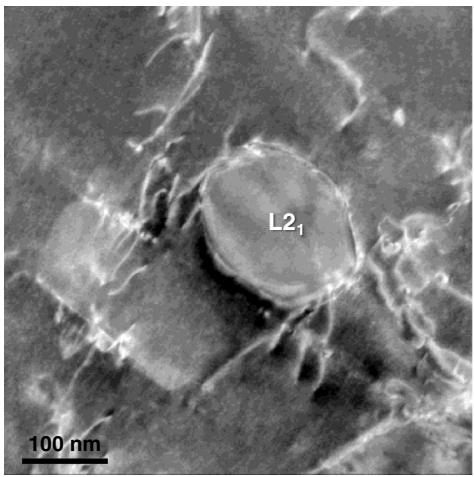

b

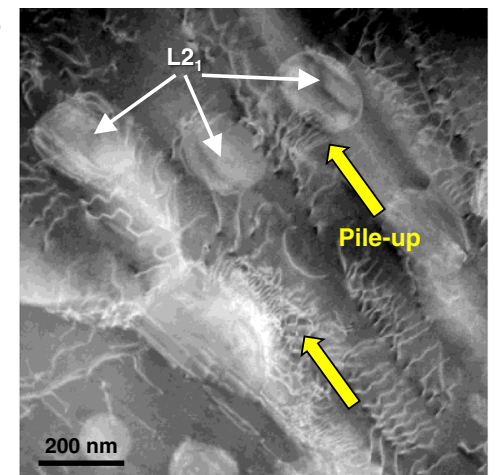

C

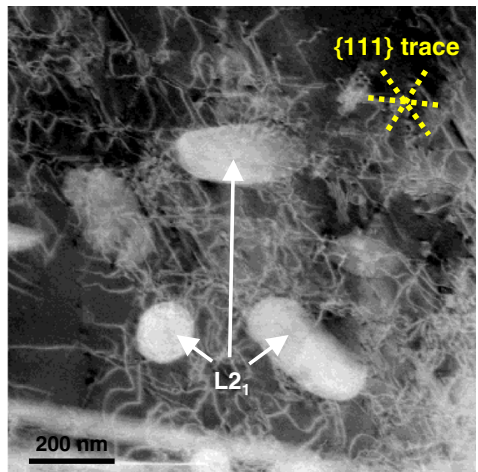

Fig. 5 High-angle annular dark-field scanning transmission electron microscopy (HAADF-STEM) images showing deformation structures for the $\mathbf{A l}_{\mathbf{0 . 2}} \mathbf{V C o N i}$ alloy annealed at $850^{\circ} \mathbf{C}$ for $\mathbf{1} \mathbf{~ h . ~ a ~ O r o w a n ~ b o w i n g ~ m e c h a n i s m ~ a t ~ a ~} L 2_{1}$ precipitate interface. $\mathbf{b}$ Dislocations pile up ahead of the $L 2_{1}$ precipitate. c Formation of planar-slip dislocation substructures along $\{111\}$ plane traces and interactions with $L_{2} 2_{1}$ precipitates. The deformation structures were observed for specimens deformed to $\sim 1 \%$ tensile strain.

mechanisms prevail. The high-angle annular dark-field scanning transmission electron microscopy (HAADF-STEM) images for the deformed A850 alloy reveal that the dislocations approaching the radial direction of cylindrical rod precipitates interact with precipitates by the Orowan bowing mechanism (Fig. 5a). On the other hand, the dislocations pile up at interfaces when they encounter precipitates of large effective radii (Fig. 5b). This pileup reduces the mean free path of dislocations, leading to significant strain hardening additionally to the Orowan bowing mechanism. For the grain subjected to a relatively large strain (Fig. 5c), the planar dislocation arrays on several independent slip planes construct dislocation network substructures and further reduce the mean free path. Therefore, this unraveled deformation mechanism has a critical role on sustaining the high strainhardening rate and delaying the necking to high strain and stress levels.

The observation of deformation structures for the specimen deformed to fracture (Supplementary Fig. 9) reveals planar slip with a high dislocation density in the recrystallized grains. This planar-slip character was also found in the equiatomic CoNiV alloy ${ }^{15}$, demonstrating that the deformation mechanism of the FCC grains additionally containing $\sim 2 \mathrm{at} \% \mathrm{Al}$ is similar to that of the CoNiV alloy. However, in non-recrystallized FCC grains, dislocation tangles are also found, although it is challenging to distinguish between the pre-existing dislocations before tensile deformation and the generated ones. Therefore, the A800 sample exhibits low ductility due to the initially high dislocation density and the limited fraction of the dislocation-free recrystallized grains. The fracture surface observations support the conclusion that the $\mathrm{L} 2_{1}$ and $\sigma$ phases do not induce brittle characteristics (Supplementary Fig. 7). Therefore, an effective strategy for achieving a proper balance between strength and ductility is to control the ratio between the recrystallized and non-recrystallized regions equivalent to the ratio between the intergranular and intragranular $\mathrm{L} 22_{1}$ precipitates, respectively. The A850 alloy exhibits a remarkable balance of mechanical properties due to the non-recrystallized regions responsible for strength and the recrystallized region responsible for ductility.

In summary, the distinctive ultrahigh strength and ductility are attributed to the microstructure decorated by finely dispersed and semicoherent nanoprecipitates along micro-shear bands. The micro-shear bands, consisting of bundles of NTs and SFs, act as heterogeneous nucleation sites and effectively hinders the formation of coarse and incoherent precipitates at grain boundaries, which have a negligible effect on strengthening. This alloy design and process route enable to modify the size, morphology, and distribution of the precipitates, leading to a remarkable strength-ductility balance of $1587 \mathrm{MPa}$ and $26.7 \%$ surpassing that of previously reported HEAs and MEAs. This work suggests a way to utilize structurally dissimilar precipitates in HEAs and MEAs, where multiprincipal elements coexist and thus can form various types of ordered intermetallic phases.

\section{Methods}

Ab initio calculations. The simulations of the phase stability were performed employing DFT calculations using the exact muffin-tin orbitals method ${ }^{35-40}$ in combination with the coherent potential approximation ${ }^{41-43}$ to account for chemical disorder. The exchange-correlation functional was approximated with the generalized gradient approximation parametrized by Perdew, Burke, and Ernzerhof ${ }^{44}$. The Brillouin zone was sampled with the Monkhorst-Pack method $^{45,46}$, where a $k$-points density of $0.1252 \pi \AA^{-1}$ was employed. The Coulomb screening parameters in the single-site approximation were fixed as $\alpha=0.75$ and $\beta=1.14$. The paramagnetic state was modeled with the disordered local moment (DLM) approximation 47,48 . The free energy differences at finite temperature were computed including the configurational entropy in the ideal mixing limit and the electronic entropy utilizing a Fermi smearing.

Alloy fabrication. An alloy of $\mathrm{Al}_{0.2} \mathrm{CoNiV}$ nominal composition (at\%) and $100 \times$ $35 \times 8 \mathrm{~mm}^{3}$ dimensions was cast using vacuum induction melting (model MC100V, Indutherm, Walzbachtal-Wossingen, Germany) and high-purity elements (>99.95\%). The blocks were homogenized at $1200^{\circ} \mathrm{C}$ for $24 \mathrm{~h}$ in evacuated quartz ampules and water quenched. The surfaces of the blocks were pickled in a $20 \% \mathrm{HCl}$ solution. Subsequently, the samples were cold-rolled until a thickness reduction of $80 \%$ was reached, and the resulting 1.5 - $\mathrm{mm}$-thick cold-rolled sheets were annealed at 800,850 , and $900^{\circ} \mathrm{C}$ in a box furnace for $1 \mathrm{~h}$ in an Ar atmosphere, followed by water quenching. To investigate the precipitation behavior excluding the shear bands, the cold-rolled sheets were annealed at $1150^{\circ} \mathrm{C}$ for $2 \mathrm{~h}$, followed by water quenching, and subsequently aged at $850^{\circ} \mathrm{C}$ for $1 \mathrm{~h}$, followed by water quenching in a box furnace at Ar atmosphere.

Microstructural characterization. XRD $\left(\mathrm{Cu} \mathrm{K}_{\alpha}\right.$ radiation, scan rate: $2^{\circ} \min ^{-1}$ scan step size: $0.02^{\circ}$ ) measurements were performed for crystallographic analyses The grain size, morphology, and distribution were investigated via EBSD and ECCI, where the specimens were prepared by mechanical polishing using a colloidal silica suspension. The EBSD measurements were performed with a field emission scanning electron microscope (FE-SEM, JEOL, JSM-6500F, USA). The average misorientation of a given point relative to its neighbors was calculated using the KAM approach. The KAM values were calculated up to the third neighbor shell with a maximum misorientation angle of $5^{\circ}$ and revealed the deformation-induced local orientation gradients. Additionally, ECCI analyses were carried out using a Zeiss-Merlin instrument (Zeiss Crossbeam 1540 EsB, Zeiss, Oberkochen, Germany). The evolved nanostructures were further investigated via TEM performed on a JEOL JEM-2100F instrument operated at $200 \mathrm{kV}$. TEM specimens were prepared using the focused ion beam (FIB) lift-out technique in a FEI Helios NanoLab 450 F1 instrument. The chemical composition of each phase was measured via APT (LEAP 4000X HR, Cameca Instruments Inc.) applying the pulsed laser mode at a specimen base temperature of $\sim 50 \mathrm{~K}$. The pulse frequency and energy were $200 \mathrm{kHz}$ and $50 \mathrm{pJ}$, respectively. The acquired APT data were reconstructed and analyzed using the commercial IVAS ${ }^{\oplus}$ software by Cameca.

Mechanical tests. Dog bone-shaped flat specimens were fabricated by electrical discharge machining. The gauge length, width, and thickness of the tensile 
specimens were $6.4,2.5$, and $1.5 \mathrm{~mm}$, respectively. Uniaxial tensile tests were carried out at room temperature using a universal testing machine (model: 8801, Instron, Canton, MA, USA) at a crosshead speed of $6.4 \times 10^{-3} \mathrm{~mm} \mathrm{~s}^{-1}$. The tensile strains were measured using a digital image correlation (DIC) system with a vision strain gauge system (ARAMIS $5 \mathrm{M}, \mathrm{GOM} \mathrm{mbH}$, Germany). Representative data were obtained by averaging three values for each datum point. To reveal the deformation structures in terms of precipitates, tensile specimens were deformed by $\sim 1 \%$ of plastic strain and observed by scanning-TEM (STEM, FEI, Talos F200X). TEM specimens were prepared via twin-jet electro-polishing in a solution containing 10 vol.\% perchloric acid and 90 vol.\% ethanol at a temperature of $-20^{\circ} \mathrm{C}$ with a potential of $20 \mathrm{~V}$. The samples tensile deformed up to fracture were investigated via ECCI.

Estimation of strengthening by various mechanisms. The yield strength of polycrystalline alloys can be obtained as a summation of the four individual contributions: intrinsic friction stress $\left(\sigma_{0}\right)$, grain-boundary strengthening $\left(\Delta \sigma_{\mathrm{GB}}\right)$, dislocation strengthening $\left(\Delta \sigma_{\rho}\right)$, and precipitation strengthening $\left(\Delta \sigma_{\mathrm{PH}}\right)$, as expressed by Eq. (1). The $\mathrm{Al}_{0.2} \mathrm{CoNiV}$ alloy has a heterogeneous microstructure composed of recrystallized and non-recrystallized regions. To quantify the contribution of each strengthening mechanism taking into account the microstructural heterogeneity, a simple rule of the mixture was used for both regions, as expressed in Eq. (2).

$$
\begin{gathered}
\sigma_{0.2}=\sigma_{0}+\triangle \sigma_{G B}+\triangle \sigma_{\rho}+\triangle \sigma_{P H} \\
\sigma_{0.2}=f_{R} \sigma_{R 0.2}+\left(1-f_{R}\right) \sigma_{N 0.2}
\end{gathered}
$$

where $f_{R}$ is the fraction of the recrystallized region and $\sigma_{R}$ and $\sigma_{N}$ are the yield strengths of the recrystallized and non-recrystallized regions, respectively. The contribution of coarse $\mathrm{L} 2$ i islands was not considered because of their low fraction, which did not significantly vary with the annealing temperatures.

In the present $\mathrm{Al}_{0.2} \mathrm{CoNiV}$ alloy, most of the $\mathrm{Al}$ added to the $\mathrm{CoNiV}$ alloy precipitated out, and only a low concentration of $\mathrm{Al}(\sim 2$ at $\%)$ remained dissolved in the disordered FCC solid-solution phase. Hence, the Hall-Petch relationship for single-phase FCC CoNiV was used to calculate the contribution of friction stress and grain-boundary strengthening, as expressed by the following equation ${ }^{49,50}$ :

$$
\sigma_{0.2}=\sigma_{0}+k d^{-1 / 2}
$$

where $k$ and $d$ represent the Hall-Petch coefficient and average grain size, respectively. The equiatomic CoNiV alloy was reported to show severe lattice distortion and consequently high friction stress of $383 \mathrm{MPa}$, which was attributed to the considerable fluctuation of the bond distance between $\mathrm{V}$ and other elements ${ }^{15}$. In addition, according to the interior source model of dislocation generation, the lattice distortion leads to both high friction stress and high Hall-Petch coefficient ${ }^{51}$. Following this model, the CoNiV alloy exhibits a high Hall-Petch coefficient of $\sim 870 \mathrm{MPa} \mu \mathrm{m}^{1 / 2}$, which implies a high sensitivity of stress to grain size.

The $\mathrm{Al}_{0.2} \mathrm{CoNiV}$ alloy specimens annealed at 800 and $850^{\circ} \mathrm{C}$ show partially recrystallized microstructures. A high density of the remaining dislocations in nonrecrystallized grains impedes dislocation motion, and thus, significantly contributes to the strengthening of the alloys. The contribution can be quantified using the well-known Taylor hardening equation (Eq. (4) $)^{52}$ :

$$
\triangle \sigma_{\rho}=M \alpha G b \rho^{1 / 2}
$$

where $M=3.06$ is the Taylor factor for the FCC crystal, $\alpha=0.2$ is a constant for FCC alloys, $G$ is the shear modulus of the CoNiV MEA, and $b$ is the Burger's vector. The average dislocation density $(\rho)$ for the non-recrystallized FCC grains, obtained from EBSD KAM analyses, is $1.77 \times 10^{15} \mathrm{~m}^{-2}$ and $1.41 \times 10^{15} \mathrm{~m}^{-2}$ for the A800 and A850 samples, respectively. The recrystallized FCC grains show dislocation densities on the order of $10^{12}-10^{13} \mathrm{~m}^{-2}$, making negligible contributions to the strength of about $20-70 \mathrm{MPa}$.

As stated above, $\mathrm{L} 2_{1}$ precipitates at the grain boundaries have little effect on the strengthening. Only the intragranular precipitates can significantly contribute to the improvement of yield strength. The intragranular $\mathrm{L} 2{ }_{1}$ precipitates, which have a different crystal structure from the FCC matrix, have a diameter of $\sim 60 \mathrm{~nm}$ in the A800 sample, indicating that it is difficult to shear the precipitates by dislocation glide. Thus, these precipitates are expected to contribute to the strengthening through the Orowan bowing mechanisms, as expressed by the following equation ${ }^{2}$ :

$$
\triangle \sigma_{P H}=M \frac{0.4 G b}{\pi(1-v)^{1 / 2}} \frac{\ln \left(\frac{2 \bar{r}}{b}\right)}{\lambda}
$$

where $v$ is the Poisson ratio, $\bar{r}$ is the average radius of the precipitates, and $\lambda$ is the average interspacing between precipitates. This equation assumes that all precipitates have an ideal spherical morphology. However, the intragranular L2 precipitates observed in this work show a rod-like morphology with a specific aspect ratio $h=c / a>1$, where a and $\mathrm{c}$ are the diameter and height of the particle, respectively. Sonderegger et al. ${ }^{53}$ modified Eq. (6) for prolate and oblate precipitates by calculating the variation in interparticle spacing according to their aspect ratio. The following equation expresses how the aspect ratio of the particle affects precipitation strengthening:

$$
\begin{gathered}
K=h^{\frac{1}{6}}\left(\frac{2+h^{2}}{3}\right)^{-\frac{1}{4}} \\
\triangle \sigma_{P H}=K^{-1} \triangle \sigma_{P H . s p h e r i c a l}
\end{gathered}
$$

where $K$ is a shape correction factor, $h$ is the aspect ratio of the particle, and $\Delta \sigma_{\mathrm{PH} \text {.spherical }}$ is the strengthening contribution of spherical particles. The aspect ratios are determined to be 3.03 and 2.83 for the A800 and A850 samples, respectively.

\section{Data availability}

The data that support the findings of this study are available from the corresponding author upon reasonable request.

Received: 26 January 2021; Accepted: 13 July 2021; Published online: 04 August 2021

\section{References}

1. Wilm, A. Physical-metallurgical investigation of aluminum-magnesium alloys. Metallurgie 8, 225-227 (1911).

2. Ardell, A. J. Precipitation hardening. Metall. Trans. A 16, 2131-2165 (1985).

3. Courtney, T. H. Mechanical Behavior of Materials. (Waveland Press, Long Grove, 2005).

4. Reed, R. C. The Superalloys: Fundamentals and Applications (Cambridge Univ. Press, Cambridge, 2008).

5. Nystrom, J., Pollock, T., Murphy, W. \& Garg, A. Discontinuous cellular precipitation in a high-refractory nickel-base superalloy. Metall. Mater. Trans. A 28, 2443-2452 (1997).

6. Belan, J. GCP and TCP phases presented in nickel-base superalloys. Mater. Today:: Proc. 3, 936-941 (2016).

7. Heckl, A., Neumeier, S., Cenanovic, S., Göken, M. \& Singer, R. F. Reasons for the enhanced phase stability of Ru-containing nickel-based superalloys. Acta Mater. 59, 6563-6573 (2011).

8. Seiser, B., Drautz, R. \& Pettifor, D. G. TCP phase predictions in Ni-based superalloys: Structure maps revisited. Acta Mater. 59, 749-763 (2011).

9. Mousavi Anijdan, S. H. \& Bahrami, A. A new method in prediction of TCP phases formation in superalloys. Mater. Sci. Eng. A 396, 138-142 (2005).

10. Yang, T. et al. Control of nanoscale precipitation and elimination of intermediate-temperature embrittlement in multicomponent high-entropy alloys. Acta Mater. 189, 47-59 (2020)

11. Kim, S.-H., Kim, H. \& Kim, N. J. Brittle intermetallic compound makes ultrastrong low-density steel with large ductility. Nature 518, 77-79 (2015).

12. Cai, Y., Lang, Y., Cao, L. \& Zhang, J. Enhanced grain refinement in AA7050 Al alloy by deformation-induced precipitation. Mater. Sci. Eng. A 549, 100-104 (2012).

13. Gludovatz, B. et al. Exceptional damage-tolerance of a medium-entropy alloy CrCoNi at cryogenic temperatures. Nat. Commun. 7, 1-8 (2016).

14. Laplanche, G. et al. Elastic moduli and thermal expansion coefficients of medium-entropy subsystems of the $\mathrm{CrMnFeCoNi}$ high-entropy alloy. J. Alloy. Compd. 746, 244-255 (2018).

15. Sohn, S. S. et al. Ultrastrong medium-entropy single-phase alloys designed via severe lattice distortion. Adv. Mater. 31, 1807142 (2019).

16. Sohn, S. S. et al. High-rate superplasticity in an equiatomic medium-entropy VCoNi alloy enabled through dynamic recrystallization of a duplex microstructure of ordered phases. Acta Mater. 194, 106-117 (2020).

17. He, J. Y. et al. A precipitation-hardened high-entropy alloy with outstanding tensile properties. Acta Mater. 102, 187-196 (2016).

18. He, J. Y. et al. Precipitation behavior and its effects on tensile properties of FeCoNiCr high-entropy alloys. Intermetallics 79, 41-52 (2016).

19. Zhao, Y. L. et al. Heterogeneous precipitation behavior and stacking-faultmediated deformation in a CoCrNi-based medium-entropy alloy. Acta Mater. 138, 72-82 (2017).

20. Liang, Y. J. et al. High-content ductile coherent nanoprecipitates achieve ultrastrong high-entropy alloys. Nat. Commun. 9, 4063 (2018).

21. Tong, Y. et al. Outstanding tensile properties of a precipitation-strengthened $\mathrm{FeCoNiCrTi}_{0.2}$ high-entropy alloy at room and cryogenic temperatures. Acta Mater. 165, 228-240 (2019).

22. Yang, T. et al. Multicomponent intermetallic nanoparticles and superb mechanical behaviors of complex alloys. Science 362, 933 (2018).

23. Choudhuri, D. et al. Crystallographically degenerate B2 precipitation in a plastically deformed FCC-based complex concentrated alloy. Mater. Res. Lett. 6, 171-177 (2018) 
24. Dasari, S. et al. Engineering multi-scale B2 precipitation in a heterogeneous FCC based microstructure to enhance the mechanical properties of a $\mathrm{Al}_{0.5} \mathrm{Co}_{1.5} \mathrm{CrFeNi}_{1.5}$ high entropy alloy. J. Alloy. Compd. 830, 154707 (2020).

25. Choudhuri, D., Shukla, S., Gwalani, B., Banerjee, R. \& Mishra, R. S. Deformation induced intermediate metastable lattice structures facilitate ordered B2 nucleation in a FCC-based high entropy alloy. Mater. Res. Lett. 7, 40-46 (2018).

26. Yang, T. et al. Effects of $\mathrm{Al}$ addition on microstructure and mechanical properties of $\mathrm{Al}_{\mathrm{x}} \mathrm{CoCrFeNi}$ High-entropy alloy. Mater. Sci. Eng. A 648, 15-22 (2015).

27. Zhang, K. \& Fu, Z. Effects of annealing treatment on phase composition and microstructure of $\mathrm{CoCrFeNiTiAl}_{\mathrm{x}}$ high-entropy alloys. Intermetallics 22, 24-32 (2012).

28. Tong, C.-J. et al. Microstructure characterization of $\mathrm{Al}_{\mathrm{x}} \mathrm{CoCrCuFeNi}$ highentropy alloy system with multiprincipal elements. Metall. Mater. Trans. A 36, 881-893 (2005).

29. He, J. Y. et al. Effects of $\mathrm{Al}$ addition on structural evolution and tensile properties of the FeCoNiCrMn high-entropy alloy system. Acta Mater. 62, 105-113 (2014).

30. Olson, G. \& Cohen, M. Kinetics of strain-induced martensitic nucleation. Metall. Trans. A 6, 791 (1975).

31. Stone, R. H. V., Cox, T. B., Low, J. R. \& Psioda, J. A. Microstructural aspects of fracture by dimpled rupture. Int. Met. Rev. 30, 157-180 (1985).

32. Li, C. et al. Microstructures and mechanical properties of body-centered-cubic $(\mathrm{Al}, \mathrm{Ti})_{0.7}(\mathrm{Ni}, \mathrm{Co}, \mathrm{Fe}, \mathrm{Cr})_{5}$ high entropy alloys with coherent $\mathrm{B} 2 / \mathrm{L} 2_{1}$ nanoprecipitation. Mater. Sci. Eng. A 737, 286-296 (2018).

33. Furuhara, T. \& Maki, T. Variant selection in heterogeneous nucleation on defects in diffusional phase transformation and precipitation. Mater. Sci. Eng. A 312, 145-154 (2001).

34. Luo, C. P. \& Weatherly, G. C. The invariant line and precipitation in a Ni-45 wt\% Cr alloy. Acta Metall. 35, 1963-1972 (1987).

35. Andersen, O. K. et al. Third-Generation TB-LMTO. Mater. Res. Soc. Symp. Proc. 491, 3 (1997).

36. Ruban, A. V., Belonoshko, A. B. \& Skorodumova, N. V. Impact of magnetism on Fe under Earth's core conditions. Phys. Rev. B 87, 014405 (2013).

37. Ruban, A. V., Simak, S. I., Korzhavyi, P. A. \& Skriver, H. L. Screened Coulomb interactions in metallic alloys. II. Screening beyond the single-site and atomicsphere approximations. Phys. Rev. B 66, 024202 (2002).

38. Ruban, A. V. \& Skriver, H. L. Screened Coulomb interactions in metallic alloys. I. Universal screening in the atomic-sphere approximation. Phys. Rev. B 66, 024201 (2002).

39. Vitos, L. Total-energy method based on the exact muffin-tin orbitals theory. Phys. Rev. B 64, 014107 (2001).

40. Vitos, L. Computational Quantum Mechanics for Materials Engineers: the EMTO Method and Applications (Springer, London, 2007).

41. Soven, P. Coherent-potential model of substitutional disordered alloys. Phys. Rev. 156, 809-813 (1967).

42. Gyorffy, B. L. Coherent-potential approximation for a nonoverlappingmuffin-tin-potential model of random substitutional alloys. Phys. Rev. B 5, 2382-2384 (1972).

43. Ruban, A. V. \& Skriver, H. L. Calculated surface segregation in transition metal alloys. Comput. Mater. Sci. 15, 119-143 (1999).

44. Perdew, J. P., Burke, K. \& Ernzerhof, M. Generalized gradient approximation made simple. Phys. Rev. Lett. 77, 3865-3868 (1996).

45. Baldereschi, A. Mean-value point in the Brillouin zone. Phys. Rev. B 7, 5212-5215 (1973).

46. Monkhorst, H. J. \& Pack, J. D. Special points for Brillouin-zone integrations. Phys. Rev. B 13, 5188-5192 (1976).

47. Staunton, J., Gyorffy, B. L., Pindor, A. J., Stocks, G. M. \& Winter, H. The "disordered local moment" picture of itinerant magnetism at finite temperatures. J. Magn. Magn. Mater. 45, 15-22 (1984).

48. Gyorffy, B. L., Pindor, A. J., Staunton, J., Stocks, G. M. \& Winter, H. A firstprinciples theory of ferromagnetic phase transitions in metals. J. Phys. F: Met. Phys. 15, 1337-1386 (1985).

49. Hall, E. O. The deformation and ageing of mild steel: III discussion of results. Proc. Phys. Soc. Sect. B 64, 747-753 (1951).
50. Petch, N. J. The cleavage strength of polycrystals. J. Iron Steel Inst. 174, 25-28 (1953).

51. Armstrong, R., Codd, I., Douthwaite, R. M. \& Petch, N. J. The plastic deformation of polycrystalline aggregates. Philos. Mag. 7, 45-58 (1962).

52. Sanaty-Zadeh, A. Comparison between current models for the strength of particulate-reinforced metal matrix nanocomposites with emphasis on consideration of Hall-Petch effect. Mater. Sci. Eng. A 531, 112-118 (2012).

53. Sonderegger, B. \& Kozeschnik, E. Particle strengthening in FCC crystals with prolate and oblate precipitates. Scr. Mater. 66, 52-55 (2012).

\section{Acknowledgements}

This work was supported by the POSCO Science Fellowship of POSCO TJ Park Foundation; the National Research Foundation of Korea (NRF-2020R1C1C1003554); the Creative Materials Discovery Program of the National Research Foundation of Korea (NRF) funded by the Ministry of Science and ICT (NRF-2016M3D1A1023384); the Korea Institute for Advancement of Technology (KIAT) grant funded by the Korea Government (MOTIE, P0002019, The Competency Development Program for Industry Specialist); Nederlandse Organisatie voor Wetenschappelijk Onderzoek (NWO)/Stichting voor de Technische Wetenschappen (STW), VIDI Grant No. 15707; and German Research Foundation (Deutsche Forschungsgemeinschaft, DFG) under the priority programme 2006 "CCA - HEA".

\section{Author contributions}

T.J.J. and W.S.C. contributed equally to this manuscript. S.S.S. designed the research. D.W.K. and T.J.J. fabricated materials and conducted mechanical tests. W.S.C., G.C., and H.J. carried out TEM and APT experiments. A.F. and F.K. performed ab initio calculations. T.J.J., W.S.C., P.C., and S.S.S. analyzed the data and wrote the manuscript. All authors reviewed and contributed to the final manuscript.

\section{Competing interests}

The authors declare no competing interests.

\section{Additional information}

Supplementary information The online version contains supplementary material available at https://doi.org/10.1038/s41467-021-25031-6.

Correspondence and requests for materials should be addressed to P.-P.C. or S.S.S.

Peer review information Nature Communications thanks Jakub Cizek and the other, anonymous, reviewers for their contribution to the peer review of this work. Peer reviewer reports are available.

Reprints and permission information is available at http://www.nature.com/reprints

Publisher's note Springer Nature remains neutral with regard to jurisdictional claims in published maps and institutional affiliations.

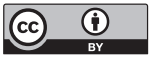

Open Access This article is licensed under a Creative Commons Attribution 4.0 International License, which permits use, sharing, adaptation, distribution and reproduction in any medium or format, as long as you give appropriate credit to the original author(s) and the source, provide a link to the Creative Commons license, and indicate if changes were made. The images or other third party material in this article are included in the article's Creative Commons license, unless indicated otherwise in a credit line to the material. If material is not included in the article's Creative Commons license and your intended use is not permitted by statutory regulation or exceeds the permitted use, you will need to obtain permission directly from the copyright holder. To view a copy of this license, visit http://creativecommons.org/ licenses/by/4.0/.

(C) The Author(s) 2021, corrected publication 2021 